\title{
Philosophiques
}

\section{Sébastien Gandon et Ivahn Smadja (dir.), Philosophie des mathématiques : logiques, preuves et pratique, Paris, Librairie philosophie J. Vrin, 2017, 384 pages}

\section{Jeffrey Elawani}

Volume 45, numéro 2, automne 2018

URI : https://id.erudit.org/iderudit/1055286ar

DOI : https://doi.org/10.7202/1055286ar

Aller au sommaire du numéro

Éditeur(s)

Société de philosophie du Québec

ISSN

0316-2923 (imprimé)

1492-1391 (numérique)

Découvrir la revue

Citer ce compte rendu

Elawani, J. (2018). Compte rendu de [Sébastien Gandon et Ivahn Smadja (dir.), Philosophie des mathématiques : logiques, preuves et pratique, Paris, Librairie philosophie J. Vrin, 2017, 384 pages]. Philosophiques, 45(2), 559-564.

https://doi.org/10.7202/1055286ar d'utilisation que vous pouvez consulter en ligne.

https://apropos.erudit.org/fr/usagers/politique-dutilisation/ 
pensée féministe et queer, restera au fil des 448 pages malheureusement muet au sujet des femmes, personnes concernées au premier plan par la procréation et le mariage.

Soulignons en terminant le défi posé par la délicate tâche d'édition menée par Frédéric Gros, qui a pris soin d'indiquer et de justifier les interventions nécessaires dans le texte et dans une très éclairante introduction. Il s'agissait de respecter la volonté des ayants droit en évitant de produire une édition critique où une glose éditoriale aurait risqué d'étouffer la voix de l'auteur par l'ajout d'un exosquelette exégétique, tout en comblant les quelques manques inévitables liés à l'absence de directives précises quant à l'emplacement de certaines sections. Le travail d'édition arrive à restituer la richesse et la fluidité de la pensée foucaldienne grâce à des divisions claires qui facilitent la préhension et la navigation dans le texte. Ainsi mise en valeur, la maniera foucaldienne permet de comprendre les fondements de certains conflits idéologiques intemporels, mais surtout de comprendre comment «sont liés, par des liens que notre culture a plutôt tendus que dénoués, le sexe, la vérité et le droit» (p. 36I).

C'est là un des rôles de l'histoire, que nous pouvons mieux saisir grâce à la lecture de l'œuvre de Foucault: nous rappeler que le passé est souvent d'une criante actualité, et que dans ses replis les plus intimes l'humain ne change guère: ses élans, besoins, certes souvent façonnés par le biopouvoir, changent peu alors que l'organisation sociale autour de lui change de visage selon les lieux et les époques.

MÉLISSA THÉRIAULT

Université du Québec à Trois-Rivières

\section{Sébastien Gandon et Ivahn Smadja (dir.), Philosophie des mathé- matiques: logiques, preuves et pratique, Paris, Librairie philosophie J. Vrin, 2017, 384 pages}

S. Gandon et I. Smadja réunissent, dans un second tome dédié à la philosophie des mathématiques, la traduction française de six publications anglophones relativement récentes ${ }^{1}$. Si le précédent recueil, "Ontologie, vérité, fondements ", adoptait une orientation classiquement philosophique sur le sujet, l'intitulé "Logique, preuve et pratique» de la nouvelle parution indique une plus grande proximité avec les mathématiques effectives.

Partagés également en trois sections, les articles sont unis par leur confrontation à une attitude qui posait traditionnellement les problèmes de philosophie mathématique dans un cadre logique et ensembliste consen-

1. Seul le texte de Tymozcko (I979) remonte à une date antérieure à I990. 
suel $^{2}$. Le présent tome porte en cela le projet du premier: celui d'introduire mathématiciens et philosophes à l'actualité de recherches originales mais ignorées du public francophone. Ses trois sections sont accompagnées de longues introductions puisque les deux présentateurs ne se cachent pas de la plus grande technicité de l'ouvrage, et de leur volonté de rendre la lecture abordable à l'amateur sérieux ${ }^{3}$.

La première des sections, qui regroupe les textes de Jamie Tappenden, "Concepts mathématiques et définition», et de Mark Wilson, "Peut-on se fier à la forme logique ? ", est introduite par une présentation de l'inspiration philosophique qui anime les deux auteurs. L'extension naturelle d'une théorie en mathématiques semble supposer que les concepts possèdent un sens propre au-delà de la stipulation logique qui les définit dans tel système formel restreint. En proposant une lecture de type contextuel de la philosophie de Frege, Tappenden et Wilson prennent acte, d'après les présentateurs, de cette "croissance conceptuelle inhérente aux mathématiques effectives ${ }^{4}$ ». Si la discussion qui s'ensuit sur l'interprétation et l'héritage frégéens des auteurs est intéressante, il est cependant douteux que son intérêt aide à une meilleure intelligence de leurs articles. Ceux-ci se résument ainsi.

Le texte de Tappenden ${ }^{5}$ traite de l'apport épistémologique des définitions en mathématique et promeut le concept de définition réelle. La réalité d'une définition vient, entre autres, de son pouvoir à saisir le bon trait explicatif dans une perspective d'approfondissement de la recherche mathématique. Par exemple, le symbole de Legendre, tel que défini en théorie élémentaire des nombres, paraît un moyen artificiel et économique, prima facie, de présenter le théorème de réciprocité quadratique. Pourtant, dans le cadre algébrique moderne, la loi de réciprocité quadratique sous la forme ramassée du symbole de Legendre est un cas particulier d'un théorème d'isomorphisme fécond entre certains groupes d'intérêts. Tappenden tire deux leçons de cet exemple: I) que la seule forme logique ne renseigne pas sur la réalité ou l'artificialité d'une définition puisque le symbole de Legendre définit en apparence, par une fonction, une propriété de type disjonctive. Or ces propriétés, par exemple "Vleu», la propriété d'être vert ou bleu, se remarquent par une forme logique qui aiguille vers leur caractère artificiel; 2) que des justifications mathématiques inhérentes sont nécessaires pour

2. Sébastien Gandon et Ivahn Smadja, «Introduction », dans Sébastien Gandon et Ivahn Smadja (dir.), Philosophie des mathématiques: logiques, preuves et pratique, Paris, Librairie philosophie J. Vrin, 20I7, p. I2.

3. Ibid., p. 22-23.

4. Sébastien Gandon et Ivahn Smadja, "Présentation. De la logique aux mathématiques", dans Sébastien Gandon et Ivahn Smadja (dir.), Philosophie des mathématiques: logiques, preuves et pratique, p. 30.

5. Jamie Tappenden, "Concepts mathématiques et définition », dans Sébastien Gandon et Ivahn Smadja (dir.), Philosophie des mathématiques: logiques, preuves et pratique, trad. Ivahn Smadja, p. 53-88. 
juger qu'une définition est réelle. Tappenden enchaîne que la découverte d'une bonne définition ne peut être réduite ni à un changement d'opinion eu égard aux propriétés de la chose définie ni à une simple convention linguistique nouvelle. Reste à avouer que la bonne définition nous fait progresser vers une meilleure connaissance de la chose définie.

Le texte de Mark Wilson ${ }^{6}$ part du fonctionnement de l'inférence mathématique pour éclaircir certains aspects du langage et du raisonnement en général. L'auteur met en avant une distinction entre grammaire standard et grammaire active. La première relève de l'analyse logico-grammaticale inculquée à un locuteur en puissance lors de son premier apprentissage. La seconde est une compréhension postérieure que l'on tire de la pratique effective du langage, et des succès de certains modes de raisonnement qui défient la grammaire apparente. Wilson donne l'exemple des propositions sur le statut de certains points qui appartiennent à des courbes algébriques. Avant la formulation moderne en termes de multiplicité, on traitait un point de multiplicité deux comme «deux points infiniment voisins». Or certaines propositions mathématiques sensées sur les points réguliers ne sont ni vraies ni fausses dans le cas des points infiniment voisins. Cela vient contraindre l'application des règles que la logique apparente étendrait à toutes les propositions sur les courbes algébriques. À partir de cet exemple, Wilson élabore, en suivant le fil de l'histoire des sciences et de la pratique linguistique ordinaire, une proposition sur l'évolution de la compréhension grammaticale de notre langage qui ne doit pas être rivée dogmatiquement à la première ébauche de logique que nous en acquérons.

La seconde section du livre porte sur la formalisation et la fondation des mathématiques et répond le mieux aux attentes communes envers la philosophie des mathématiques. Cette fois, la présentation des articles qui la composent, ceux de Steward Shapiro, "La logique de second ordre, les fondements et les règles", et de Solomon Fefferman, "Qu'est-ce qui repose sur quoi ", fournit une aide précieuse à l'amateur. Au bénéfice de celui-ci, elle introduit le concept de catégoricité et discute du théorème de Löwenhein-Skolem. Elle donne ensuite trois arguments favorables à une formalisation de second ordre des mathématiques, qui éclairent lumineusement le raisonnement wittgensteinien de Shapiro sur l'application d'une règle mathématique.

Afin d'introduire au texte touffu de Fefferman, Gandon et Smadia enchaînent avec une évaluation des conséquences des théorèmes d'incomplétude pour l'entreprise de fondation finitiste. Ils présentent, entre autres, une distinction fort intéressante de la non-prouvabilité en termes de logique classique et de la non-prouvabilité en termes de logique finitiste. L'introduction de la seconde section se termine par une brève étude de définitions

6. Mark Wilson, «Peut-on se fier à la forme logique? ", dans Sébastien Gandon et Ivahn Smadja (dir.), Philosophie des mathématiques: logiques, preuves et pratique, trad. Ivahn Smadja, p. $89-\mathrm{I} 33$. 
rigoureuses du finitisme et par l'exposition d'un programme de Hilbert révisé sous l'effet des résultats de Gödel. On peut rendre compte ainsi des deux textes de cette section.

Steward Shapiro ${ }^{7}$ défend la logique de second ordre contre l'affirmation que la quantification des variables de prédicats, interprétés comme sous-ensembles quelconques d'un domaine, demande une clarification au moyen d'une méta-théorie du premier ordre sur la sémantique du second ordre. En effet, Shapiro tente de montrer comment les problèmes sur le parcours des variables de prédicat se posent à nouveau dans cette méta-théorie et, plus généralement, dans toutes les théories mathématiques traitant de l'infini. La meilleure conduite est donc de prendre acte de cette quantification fort répandue sur les sous-ensembles. Dès lors, la logique de second ordre, comme formalisation des mathématiques, présente deux avantages. D'abord, puisque le théorème de Löwenhein-Skolem ne tient pas pour les théories d'ordre supérieur au premier, il n'est pas impossible d'y caractériser des structures mathématiques à un isomorphisme près. Il y a un sens naturel à parler de modèle standard, et on y entend des expressions chères aux mathématiciens sans besoin de clarification supplémentaire aboutissant à une vaine régression. Ensuite, en quantifiant sur des sous-ensembles du domaine, ce langage offre, en certaines occasions, un axiome unique où un langage de premier ordre présente un schéma d'axiomes particuliers. Or, quand «les ressources expressives sont élargies ${ }^{8}$ », ce dernier schéma produit de nouveaux axiomes, et donc, une nouvelle théorie, alors que le mathématicien poursuit pour sa part son raisonnement comme s'il appliquait simplement le même axiome de la même théorie à de nouveaux cas?

Dans l'article suivant, "Qu'est-ce qui repose sur quoi » écrit par Fefferman ${ }^{10}$, il est question de réduction théorique dans le sillage des théorèmes d'incomplétude de Gödel. Des systèmes arithmétiques au pouvoir d'expression varié sont considérés. En examinant formellement les résultats de la réduction démonstrative de systèmes justifiés par des hypothèses philosophiques «fortes » (par exemple, par l'hypothèse de l'infini actuel) à des systèmes de fondation plus modérée (en contrepartie, par l'hypothèse finitiste), Fefferman propose une version adoucie du programme de Hilbert. Ces résultats de la théorie de la démonstration, dont il explique le vocabulaire et pour lesquels il définit en termes arithmétiques le concept de réduction, doivent démontrer rigoureusement quels sacrifices mathématiques

7. Stewart Shapiro, «La logique du second ordre, les fondements et les règles », dans Sébastien Gandon et Ivahn Smadja (dir.), Philosophie des mathématiques: logiques, preuves et pratique, trad. Jean-Baptiste Rauzy, p. I73-2 I6.

8. Ibid., p. 2 I3.

9. Shapiro donne l'exemple de deux modèles de l'analyse réelle (Ibid., p. 210).

10. Solomon Fefferman, "Qu'est-ce qui repose sur quoi ?", dans Sébastien Gandon et Ivahn Smadja (dir.), Philosophie des mathématiques: logiques, preuves et pratique, trad. Jean Fichot, p. 217-256. 
sont logiquement impliqués par l'adoption d'une hypothèse philosophique déterminée.

La dernière section contient deux textes consacrés à des théories mathématiques particulières: la théorie des catégories que Steve Awodey met à contribution pour repenser la philosophie structuraliste dans «Les structures en mathématiques et en logique », et la théorie des graphes dont un des théorèmes est repris par Thomas Tymoczko dans «Le problème des quatre couleurs» afin d'interroger l'opposition traditionnelle des mathématiques aux sciences expérimentales. La présentation par Sylvain Cabanacq ${ }^{11}$ se compose d'un historique de la théorie catégorique, d'une explication redondante de la preuve des quatre couleurs - analysée très clairement par Tymoczko lui-même - et d'une courte discussion sur la réception des deux articles, lesquels vont comme suit.

Dans son article, Awodey ${ }^{12}$ développe un concept adéquat de structure mathématique dans le but d'élucider la position structuraliste en philosophie des mathématiques. Il emploie à cet effet la théorie des catégories. La première partie de son article propose d'abord une introduction au langage des catégories. Il y est ensuite question de la plus grande facilité avec laquelle cette théorie réussit à formaliser le concept de propriété structurale sans qu'interviennent, dans cette formalisation, des déterminations indépendantes de la structure des objets d'intérêt. On définit, selon la catégorie, les propriétés de ses objets au moyen des morphismes qui les concernent. Les propriétés structurales sont celles que conservent les isomorphismes. Réflexivement, les catégories sont susceptibles du même traitement catégorique structural en termes de morphismes. On parle de foncteurs dans le cas de ces derniers. Ce traitement réflexif est essentiel, il nous semble, pour éclairer comment est recevable la définition donnée par Awodey, qui, pour définir leur identité de structure, part de l'observation que deux objets de même structure appartiennent formellement à la même catégorie ${ }^{13}$.

Dans la seconde partie de son article, Awodey explique l'étude catégorique de la logique par la théorie des topoi. Cette étude partage certaines propriétés de la logique de Russell, dans laquelle les idées de fonction propositionnelle et de type jouent un rôle central. Un théorème de correspondance la rend par ailleurs plus élégante. Il est possible de lier une théorie logique à des propriétés structurales au sens de la théorie des catégories. La théorie des ensembles, par exemple, peut être doublement caractérisée par une certaine logique au sens des topoi, bivalente et tiers-exclusive, et par les

11. Sylvain Cabanacq, "Présentation. Perspectives", dans Sébastien Gandon et Ivahn Smadja (dir.), Philosophie des mathématiques: logiques, preuves et pratique, p. 259-285.

12. Steve Awodey, "Les structures en mathématiques et en logique", dans Sébastien Gandon et Ivahn Smadja (dir.), Philosophie des mathématiques: logiques, preuves et pratique, trad. Brice Halimi, p. 287-322.

13. Ibid., p. 297-298. 
propriétés des morphismes impliqués. Awodey évoque pour finir une interprétation structurale d'une fondation particulière des mathématiques, la fondation ensembliste, même si cette fondation ne fait pas partie du projet structuraliste.

Clôturant ce recueil, le texte de Tymozcko ${ }^{14}$ remet en cause le caractère apriorique des mathématiques à la faveur de la démonstration du théorème des quatre couleurs par Appel et Haken. Un problème philosophique découle de cette démonstration. En effet, celle-ci exige l'application par ordinateur d'un algorithme qui vérifie que les éléments d'un ensemble de sous-graphes satisfont une certaine propriété de réductibilité. La taille des sous-graphes rend cette application inévitable. De plus, Tymozcko défend l'improbabilité d'une preuve de nature différente, sans recours informatique. Ainsi, l'unique démonstration suppose que la vérification de la réductibilité par un programme s'effectue conformément à une certaine suite d'instructions, et que cette vérification a été effectivement conduite par un ordinateur. Or ce dernier point est un résultat empirique.

Concluons que le tour d'horizon cerné par ce recueil est de grande ampleur et initie le lecteur amateur à une variété de recherches que l'opinion courante relève trop peu. Le travail de présentation est minutieux et rigoureusement informé quand bien même il semble parfois inopportun. Quant aux textes, bien que celui de Tymozcko donne une caractérisation vague et déplorable du raisonnement mathématique, ils proposent pour le reste des études subtiles, circonspectes et profondes de la pratique mathématique.

JEFFREY ELAWANI

Université de Montréal

\section{Guillaume Lejeune, Hegel anthropologue, Paris, CNRS Éditions, 2016, 200 pages}

La visée initiale de Hegel anthropologue, de Guillaume Lejeune, est assez ambitieuse: penser avec Hegel les défis contemporains liés notamment au transhumanisme tout en résolvant l'énigme anthropologique dans l'œuvre systématique de Hegel. Le résultat sera au final plus modeste sans être moins intéressant pour autant.

Néanmoins, l'ampleur des enjeux que l'auteur entend soulever dès son avant-propos est telle que les pistes de réponse suggérées tout au long de l'ouvrage et culminant dans la conclusion s'avéreront forcément schématiques et par trop générales. L'auteur en a d'ailleurs conscience lorsqu'il écrit en conclusion: «D'aucuns pourraient être déçus de la généralité de nos

14. Thomas Tymozcko, «Le problème des quatre couleurs », dans Sébastien Gandon et Ivahn Smadja (dir.), Philosophie des mathématiques: logiques, preuves et pratique, trad. Baptiste Mélès, p. 323-364. 